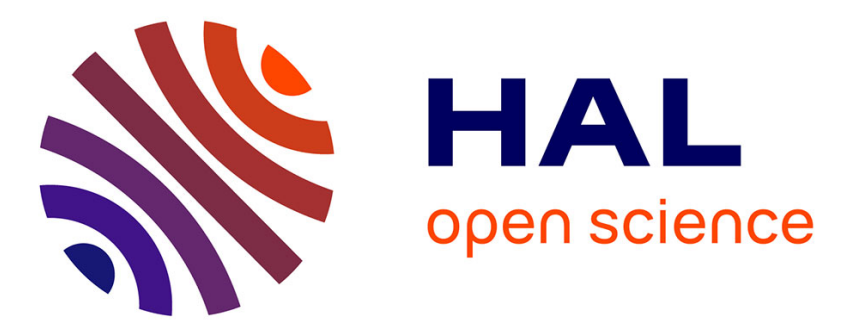

\title{
Computational Modeling of Exciton Localization in Self-Assembled Perylene Helices: Effects of Thermal Motion and Aggregate Size
}

\author{
Alekos Segalina, Xavier Assfeld, Antonio Monari, Mariachiara Pastore
}

\section{To cite this version:}

Alekos Segalina, Xavier Assfeld, Antonio Monari, Mariachiara Pastore. Computational Modeling of Exciton Localization in Self-Assembled Perylene Helices: Effects of Thermal Motion and Aggregate Size. Journal of Physical Chemistry C, 2019, 123 (11), pp.6427-6437. 10.1021/acs.jpcc.9b00494 . hal-03445152

\author{
HAL Id: hal-03445152 \\ https://hal.science/hal-03445152
}

Submitted on 23 Nov 2021

HAL is a multi-disciplinary open access archive for the deposit and dissemination of scientific research documents, whether they are published or not. The documents may come from teaching and research institutions in France or abroad, or from public or private research centers.
L'archive ouverte pluridisciplinaire HAL, est destinée au dépôt et à la diffusion de documents scientifiques de niveau recherche, publiés ou non, émanant des établissements d'enseignement et de recherche français ou étrangers, des laboratoires publics ou privés. 


\section{Computational Modelling of Exciton Localization in}

\section{Self-Assembled Perylenes Helices: Effects of Thermal}

\section{Motion and Aggregates Size}

Alekos Segalina, ${ }^{a}$ Xavier Assfeld, ${ }^{a}$ Antonio Monari, ${ }^{a}{ }^{*}$ and Mariachiara Pastore $*^{a}$

anniversité de Lorraine \& CNRS, Laboratoire de Physique et Chimie Théoriques (LPCT) UMR 7019, F-54000 Nancy, France

*e-mail: antonio.monari@univ-lorraine.fr, mariachiara.pastore@univ-lorraine.fr 


\begin{abstract}
Effects of aggregation on excited states properties in solution of perylene diimide self-assembled helixlike structures of different size are investigated by means of first principle Density Functional Theory (DFT), Time-Dependent DFT (TD-DFT) and classical Molecular Dynamic (MD) simulations. Excited state analysis based on the 1-particle transition density matrices is then used to study the exciton nature and its delocalization as a function of the thermal motion and aggregate size. Overall the results point to a rather small delocalization of the Frenkel excitonic state even in large aggregates also related to a concerted motion of blocks of 4 monomers along the MD trajectories. Although dynamic effects do not remarkably affect the calculated position and shape of the absorption spectrum, they cause the appearance of several low-energy states of charge transfer character, and hence of weak intensity (dark states), that might be populated along the ultrafast exciton relaxation process potentially influencing the charge separation processes in PDI-sensitized photoactive heterointerfaces.
\end{abstract}

\title{
Introduction
}

Organic conjugated materials have received an increasing scientific interest due to the peculiar tunable photophysical properties of their self-assembled aggregates, making them suitable for several applications, ranging from display technologies ${ }^{1,2}$ to photovoltaics devices. ${ }^{3-5}$ As a matter of fact, the intermolecular interactions taking place in supramolecular aggregates depend on the packing geometries and directly determine the optical and photophysical response, as argued for the first time by Kasha in the sixties. ${ }^{6,7}$ Kasha's model, indeed, predicts the variation of the photophysical properties of molecular aggregates, with respect to the monomer, relying solely on geometric considerations and on the change in the coupling between the respective transition dipole moments. ${ }^{6,8}$ Indeed, in a simple dimeric aggregate a blue shifted absorption maximum and a weaker radiative decay rate, compared to the monomer, are predicted when the aggregation geometry is "side-by-side" (H aggregate), while in the case of an "head-to-tail" arrangement (J aggregate) the absorption maximum is red shifted and a more 
important radiative decay rate is obtained. ${ }^{9,10}$ Moreover, formation of self-assembled monolayers of organic molecules on semiconductor surfaces has been largely exploited in photocatalysis and photovoltaic devices, being surface functionalization at the heart of the functioning of dye-sensitized photoelectrodes in Grätzel solar cells (DSCs) ${ }^{11-17}$ and photoelectrosynthesis cells (DS-PECs) for water splitting. ${ }^{18-20}$ Dye-aggregation on the metal oxide surface is, however, generally regarded as responsible for low cell efficiencies ${ }^{21-25}$ and thus prevented by the use of antiaggregation coadsorbents. ${ }^{26-28}$ On the other hand, some example of beneficial aggregation effects (higher photocurrents) have been reported, mainly related to the extension of the spectral window of the harvested light. ${ }^{29-32}$ Moreover, successful examples of exploitation of perylene diimide-based aggregates (PDI in Figure 1) in dye-sensitized photoanodes for water oxidation have been recently demonstrated. ${ }^{33-35}$ Indeed, spontaneous adsorption of $\pi$-stacked face-to-face $\mathrm{H}$-aggregates on $\mathrm{TiO}_{2}, \mathrm{WO}_{3}$ and $\mathrm{SnO}_{2}$ surfaces was evidenced by the intensity reversal between the $0 \rightarrow 0$ and $0 \rightarrow 1$ vibronic bands in the optical absorption spectrum of the PDIsensitized film compared to the one registered in low concentration solution. ${ }^{34,36,37}$

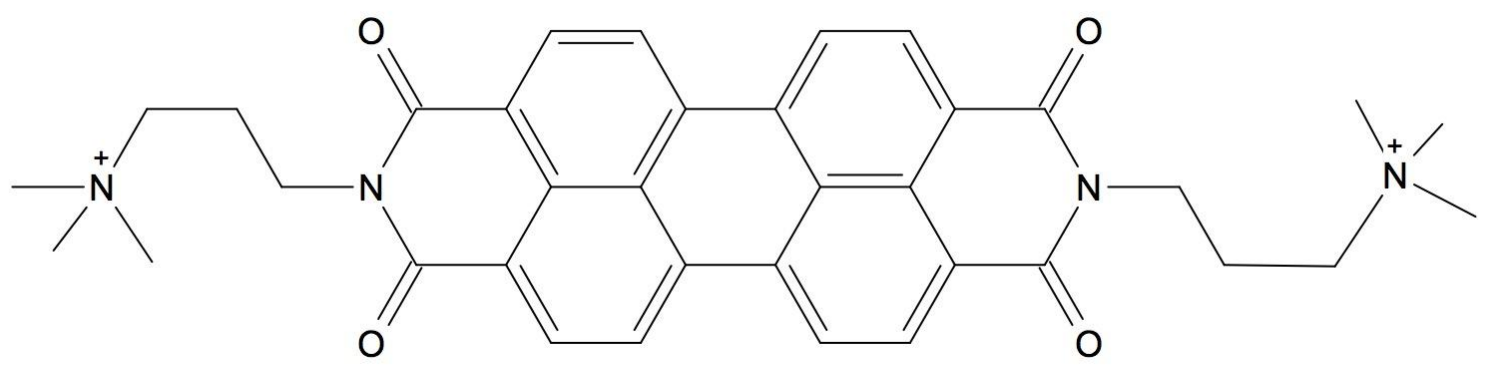

Figure 1. Chemical Formula of the PDI monomer dication.

As a matter of fact, perylenes-based chromophores have been extensively employed as photoactive materials both in bulk heterojunctions solar cells and DSCs in virtue of their exceptional chemical, thermal and photochemical stability, wide and intense optical absorption in the visible to near-infrared spectral region and good charge transport properties. ${ }^{38-40}$ PDI-based dye sensitizers, however, despite 
their systematic modification with various anchoring and electron donor groups, have delivered only rather limited efficiencies in DSCs devices, mainly because of the lack of strongly directional charge transfer from the molecule towards the semiconductor surface. ${ }^{40}$ Moreover in water oxidation devices, as shown in Ref. ${ }^{33}$, surface sensitization by hydrophobic aggregates, adsorbed on the surface by electrostatic and dispersion interactions, does not trigger the hydrolytic cleavage ${ }^{41}$ generally observed for conventional (carboxylic or phosphonic) (2, $43^{4}$ anchoring groups. On the other hand, however, to have an efficient electron/hole injection by these photoexcited aggregates into the semiconductor/catalyst, the nature (localized Frenkel-type or charge transfer (CT)-like) and delocalization (i.e. exciton length) of the exciton, as well as its sub-picosecond photo-physics are crucial, provided that the dye/metal oxide/catalyst energy levels are properly aligned. ${ }^{33,44,46}$

Exciton diffusion dynamic, excimer formations and self-trapping processes in ordered perylene aggregates have been largely investigated by both computational and experimental methods over the last decades. ${ }^{10,47,56-59,48-55}$ Despite the complex photophysics of these aggregates, there is evidence that both localized excimers and low-energy CT states participate to the excited state relaxation dynamics, with a crucial role of the packing geometry in driving the process. ${ }^{50,55,60-63}$ Much less is known on the nature and delocalization of the Frenkel-type excitonic state, due to its weak absorbance and florescence yield, making its dynamic extremely challenging to be followed experimentally. ${ }^{64,65}$ Recent femtosecond fluorescence upconversion spectroscopy measurements indicated, however, a modest delocalization of the primarily excited Frenkel state, involving about 3 monomeric units in columnar aggregates composed of 10 perylene molecules, ${ }^{64}$ and suggesting the synthesis of rather rigid co-facially aggregates structures as a way to increase coherent transport of more delocalized exciton states.

In this contribution we model and simulate the self-assembling and optical properties of perylene diimide helix-like aggregates of different size in solution and at room temperature, by combining first principle Density Functional Theory (DFT), Time-Dependent DFT (TD-DFT), 1-particle transition density matrix analysis of the excited states and classical Molecular Dynamic simulations. Our multiscale protocol aims at getting detailed insights on the exciton nature and delocalization as a 
function of the thermal/vibrational motion and the aggregate size. In line with experimental observations, ${ }^{64}$ our results reveal a moderate delocalization of the Frenkel excitonic state in disordered (“300 K”) aggregates, mostly involving 3-4 units, even in larger helices. Moreover, as a consequence of this modest delocalization of the Frenkel bright state and of a strong aggregation, always yielding a concerted motion of blocks of 4 PDIs along the MD, finite temperature effects have a negligible impact on the calculated position and shape of the absorption spectrum. On the contrary, thermal motion favors the appearance of a plethora of weak-to dark highly charge separated low-energy states that are potentially involved in the ultrafast exciton relaxation dynamics. ${ }^{61}$

\section{Computational Details and Methodology}

To provide a balanced description of both the excited states manifold of self-aggregate PDI and of the effects of the thermal evolution we set up a multiscale approach involving both quantum and classical molecular modeling simulations as detailed below.

\subsection{Static quantum chemistry calculations}

First the PDI monomer and the small size aggregates (up to 4 units) have been studied using density functional theory (DFT) to obtain the ground state equilibrium geometry, including the performance of a conformational search. On top of equilibrium geometries, the absorption spectrum has been simulated at time-dependent DFT (TD-DFT) level of theory. Geometry optimization of the PDI monomer and its aggregates (dimer, trimer and tetramer) were carried out using the $\mathrm{M} 062 \mathrm{X}^{66} / 6-31 \mathrm{G}^{*}$ level of calculation coupled to the conductor-like polarizable continuum model $(\mathrm{C}-\mathrm{PCM})^{67}$ to take into account the solvation effects (ACN and water were considered). The calculations of the excitation energies for the monomer were carried out by TD-DFT with different functionals (PBE0, B3LYP, MPW1K, M06-2X, CAM-B3LYP) in order to calibrate the level of calculation by comparison with the available experimental data. In the case of the aggregates, only the hybrid B3LYP and the long-range corrected CAM-B3LYP functionals have been considered. The first 10, 20, 30, and 40 vertical excitation energies 
have been obtained for the monomer, dimer, trimer, tetramer and heptamer, respectively. To allow a better comparison with experimental results the simulated spectra have been obtained by convoluting the vertical transition with Gaussian functions having Full Width at Half Maximum (FWHM) of $0.2 \mathrm{eV}$. All static calculations have been performed using the Guassian09 suite of code. ${ }^{68}$

\subsection{Molecular Dynamics}

Classical molecular dynamics (MD) simulations have been performed, for both the PDI monomer and the aggregates ( 4 and 7 units) to explore the conformational space of the ground state. On top of the MD trajectories statistically independent snapshots have been extracted to calculate the excited state manifold and hence simulate the absorption spectrum as the convolution of the individual vertical transitions.

The generalized amber force field $(\mathrm{GAFF})^{69}$ was used to describe the PDI potential, the point charges have been reparameterized to fit the restricted electrostatic potential (RESP), solvent molecules have been described by the TIP3 $\mathrm{P}^{70}$ model for water molecules, in addition PDI being charged counteranions $\left(\mathrm{Cl}^{-}\right)$have been added to ensure electroneutrality. The systems were set up starting from the QM optimized geometries of the monomers and aggregates, with the exception of heptamer case for which the system was built by replicating QM optimized geometrical arrangement of the PDI dimer. The simulation boxes were composed of the solute and 2870, 4130, and 5166 water molecules for the PDI monomer, tetramer, and heptamer, respectively. Periodic boundary conditions (PBC) have been used throughout and all the simulations have been performed with the Amber code and its GPU extension. ${ }^{71}$ After an initial energy minimization, carried out for 10000 cycles to remove bad contacts, followed by equilibration and thermalization, conducted for $0.4 \mathrm{~ns}$ and $0.2 \mathrm{~ns}$, respectively, production run of $0.2 \mu \mathrm{s}$ for PDI monomer and $1 \mu$ s for PDI aggregates were executed in the NPT ensemble at 1 atm and $300 \mathrm{~K}$. Newton equations of motion were solved considering a time step of $0.2 \mathrm{fs}$, using the RATTLE algorithm to fix the bond distances involving hydrogen atoms. 


\subsection{Thermal-averaged absorption spectra calculations}

From the monomer, tetramer and heptamer MD trajectories 100 conformations of the solute were extracted, equally spaced in terms of time, to perform excited states calculations using the Amber/Terachem interface. ${ }^{72}$ To save computational time, especially in the case of the large heptamer aggregates, the peripheral trimethylammonium ethylene chains were omitted and the dangling bonds were saturated with a Hydrogen atom using the link atom scheme approach. Vertical excitations were obtained at TD-DFT level of theory using the Tamm Dancoff Approximation (TDA) and a 6-31G* basis set. In the case of the monomer both B3LYP and CAM-B3LYP functionals have been employed, whereas in the case of the aggregates only CAM-B3LYP has been used to avoid the underestimation of the excitation energy of long-range charge transfer (CT) states when using non long-range corrected functionals. ${ }^{73}$ Solvation effects (water) were accounted for via a continuum polarization approach, namely the conductor like screening model $(\mathrm{COSMO})^{74}$ as implemented in Terachem. The calculated thermal averaged absorption spectra were reproduced through a convolution with Gaussian functions having FWHM of $0.1 \mathrm{eV}$.

\subsection{Excited State and exciton analysis}

To unravel the topological characteristic of the different excited states, we performed a decomposition of the one-particle transition density matrix (1TDM) following the procedure implemented in the TheoDORE 1.7 package ${ }^{75-77}$. TheoDORE performs a one-to-one mapping of the 1TDM with a general exciton wave function $\chi\left(r_{H}, r_{E}\right)$, where $r_{H}$ stands for the position operator of the hole and $r_{E}$ of the particle (electron). Following a preliminary, and in large measure arbitrary, partition of the chemical space of the (multi)chromophore systems in terms of monomeric units it is hence possible to obtain important quantitative indexes describing exciton delocalization and charge separation. In particular, we will define the charge transfer (CT) indices that may assume values comprised between 0.0 , in case of purely local excitation, and 1.0, indicative of important charge separation. Note that, since our calculations have been performed in a TD-DFT (TDA) framework, and hence only single-excitations 
are taken into account, CT of 1.0 will be representative of a total charge-separation with no spatial overlap between the hole and particle densities. On the same way, the 1TDM may be decomposed in terms of the participation ratio (PR) index that roughly gives the number of monomeric units over which the exciton wavefunction is delocalized, and hence gives a direct measure of the exciton length. For our application the partition of the chemical space was straightforward, and indeed each PDI monomer has been considered as a monomeric unit. Hence, PR will directly give the number of PDI units over which the electronic density is delocalized. If for simplicity we consider the dimer case PR close to 2.0 and CT close to 0.0 will represent a pure Frenkel delocalized exciton.

\section{DFT Analysis of the structural and optical properties of PDI}

\section{aggregates}

To preliminarily calibrate the level of calculation, the vertical $\left(\mathrm{E}_{\max }\right)$ and adiabatic $\left(\mathrm{E}_{0-0}\right)$ excitation energies of the PDI monomer in ACN and water were calculated using different exchange and correlation functionals and compared to the experimental values; the results are reported in Table S1 in Supporting Information. The lowest energy bright state of PDI is described by a single electronic excitation from the HOMO to the LUMO, that are localized on the perylene's conjugate core; as

expected, no involvement of the lateral charged chains (trimethylammonium - ethylene) is observed. ${ }^{78}$ As a first important point, if we compare the $E_{0-0}$ energies obtained from the TD-DFT calculations in water and those obtained in ACN we can underline the modest or even negligible solvatochromism observed for those systems. This will in turn justify our choice to perform the subsequent calculations involving MD simulations in water due to the much better availability of good force field parameters for the latter than for ACN. On the other hand, the good agreement between the experimental value of $E_{0-0}$, and the one calculated with the PBE0 functional also stands out. In the case of vertical excitation energies B3LYP appears as the outperforming functional; hence globally and, as expected, the hybrid functionals incorporating a moderate fraction of non-local exchange are by far the most appropriate ones 
to model optical properties of PDI, i.e. paradigmatic $\pi$-conjugated organic systems. ${ }^{62}$ Furthermore, since B3LYP was also performing rather satisfactorily concerning the value obtained for the adiabatic excitation energies, we decided to systematically use this functional for the following study and the topological characterization of the monomers.
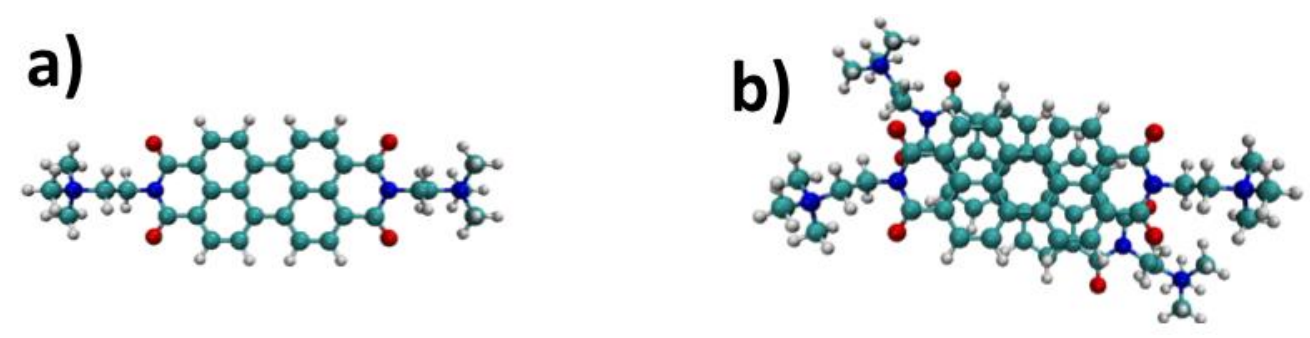

Figure 2. Optimized structure of PDI monomer (a), dimer (b)

The ab-initio equilibrium geometries obtained for the PDI aggregates, namely dimer and trimer, are reported in Figure 2. To find the most stable dimeric configuration, a relaxed dihedral angle scan between two PDI molecules has also been performed and the calculated energy profile is shown in Figure S1. As elsewhere reported for analogous perylenes, the total energy minimum for the stacked PDI dimer was found for a dihedral angle of about 30 degrees between the PDI units. ${ }^{36}$ Thus, the trimer was built starting from the optimized dimer geometry and placing the third unit at 30 or -30 degrees with respect to the adjacent molecule, building the so called "alternated type" and the "helix like" aggregate, respectively (Figure S2). The two conformations were found to be quasi-degenerate, since the "helix-like" structure resulted to be only $0.37 \mathrm{Kcal} / \mathrm{mol}$ below the "alternated type" one. In the case of the tetramer the "helix like" and the "alternated type" aggregates were manually constructed and then fully optimized; once again, the former conformation appears to be more stable by about $1.52 \mathrm{Kcal} / \mathrm{mol}$. Obviously, this quasi-degeneracy is indicative of a significant Boltzman population of both states and conformers. However, as we shall discuss later, the coexistence of the two conformations has a negligible influence on the position of the absorption maximum. Finally, the distance between the center of mass of the PDI monomers was calculated to be ca. $3.5 \AA$, in good agreement with the values expected for $\pi$-stacked systems. ${ }^{36,79}$ 
In order to quantify the magnitude of the forces leading to self-organization in the ground state, the binding energies were calculated for the fully relaxed aggregates in "helix like" geometries. In Figure S3 the normalized binding energy per unit is reported versus the number of monomer. An increase in the normalized binding energy for the larger systems is evident, hence showing a non-additive and cooperative stabilization, which appears to start reaching a plateau for the tetramer aggregate. The per unit binding energies are comprised between $14.90 \mathrm{Kcal} / \mathrm{mol}$ (dimer) and 22.14Kcal $/ \mathrm{mol}$ (tetramer).

From the respective equilibrium geometries, vertical excitation energies were computed for the aggregates, also considering both possible conformations in the case of trimer, tetramer and heptamer.

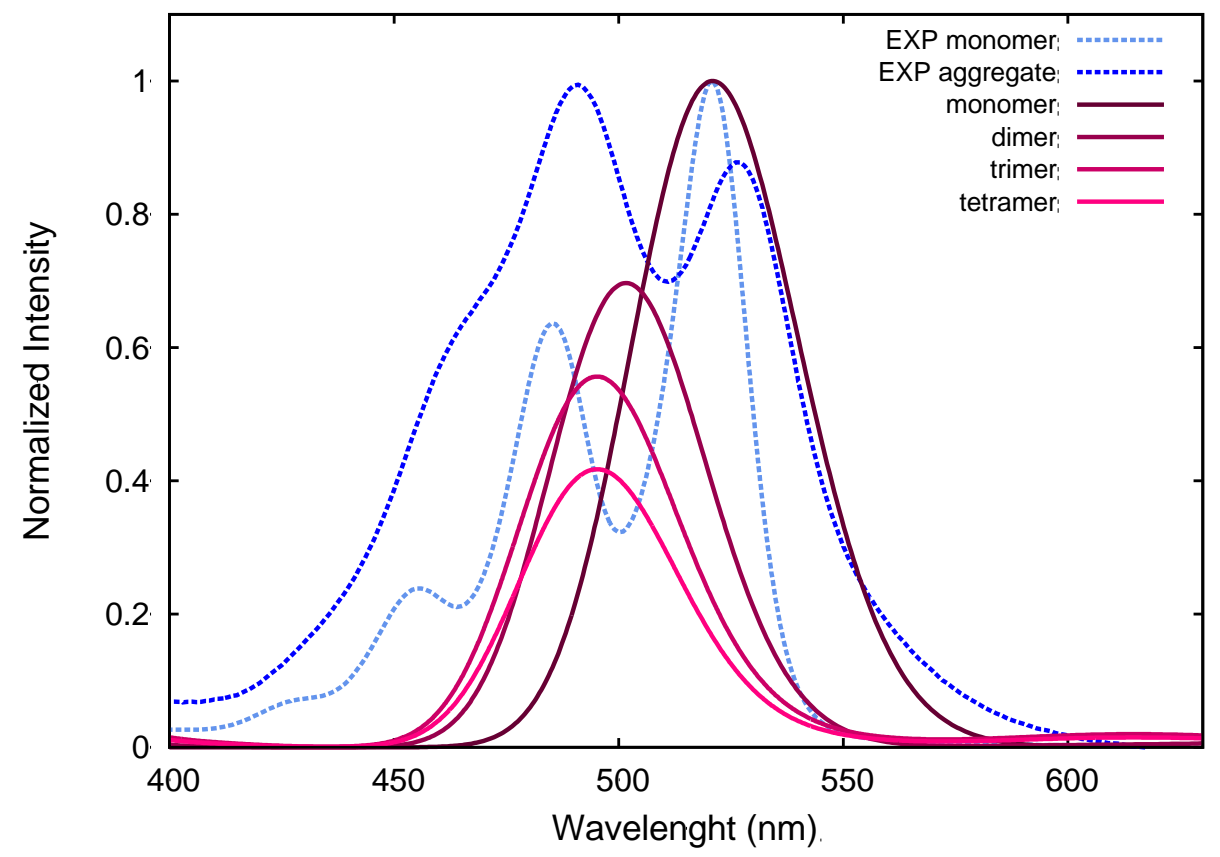

Figure 3. Calculated (full lines) and experimental (dashed lines) absorption spectra of the PDI monomer and "helix like" aggregates. The calculations have been performed at B3LYP/6-31G* level of theory in implicit (C-PCM) water.

The convoluted spectra up to the tetramer are displayed in Figure 3 compared with the experimental ones, while the corresponding vertical excitation energies of the bright states and their oscillator strengths are listed in Table 1, along with the experimental absorption maxima and the calculated CT number and PR indices. In order to check on the convergence of the aggregates' optical properties, we 
also added in Table 1 the calculated vertical excitation energies at the TDA/COSMO level of theory (see section 2.3) for the heptamer aggregates optimized using the amber FF. Note that in Figure 3, the "helix like" geometries for the trimer and tetramer were arbitrarily chosen, given the negligible differences in the position of the calculated absorption maxima (Table 1), amounting to $0.02 \mathrm{eV}$ at most in the case of the trimer. In Figure 3, it is possible to evidence the blue shift and the decrease of the oscillator strength (hypochromism) from the monomer to the larger aggregates that are typical features observed in the case of $\mathrm{H}$ aggregates. ${ }^{6,80,81}$ Moreover, a good agreement between the absorption maxima of the experimental spectrum of the aggregate (blue dashed line) and those of trimer (violet full line) and tetramer (magenta full line) also appears, as confirmed by the data in Table 1. The calculated excitation energies for the two heptamers result sizably blue shifted when compared to the ones obtained on the tetramer. This shift at higher energies, comes from the level of calculation employed (TDA) rather than from an electronic effect related to the increased size, as it will be discussed in section 4 and shown in Table S2 in Supporting Information. Also, the computed oscillator strengths at TDA level of theory are not comparable to the ones obtained from the TDDFT calculations. We, thus, consider these results on the heptamer aggregates only to gain information about the trend of the CT and PR indices as the size of the aggregate increases. 


\begin{tabular}{|c|c|c|c|c|}
\hline System & $\begin{array}{l}E_{\max } \\
(\mathrm{eV})\end{array}$ & Oscillator Strength & CT numbers & $\begin{array}{c}\text { Participation ratio } \\
\text { (PR) }\end{array}$ \\
\hline monomer $^{\mathrm{a}}$ & 2.38 & 0.902 & - & - \\
\hline dimer $^{a}$ & 2.47 & 1.340 & 0.035 & 2.00 \\
\hline \multicolumn{5}{|c|}{ Helix like } \\
\hline trimer $^{a}$ & 2.50 & 1.096 & 0.216 & 2.17 \\
\hline tetramer $^{\mathrm{a}}$ & 2.54 & 1.235 & 0.236 & 3.21 \\
\hline \multirow{2}{*}{ heptamer $^{\text {b }}$} & 2.72 & 3.753 & 0.125 & 4.98 \\
\hline & 2.77 & 2.429 & 0.118 & 5.76 \\
\hline \multicolumn{5}{|c|}{ Alternated type } \\
\hline trimer $^{a}$ & 2.52 & 1.667 & 0.075 & 2.50 \\
\hline tetramer $^{\mathrm{a}}$ & 2.55 & 2.127 & 0.072 & 3.53 \\
\hline heptamer $^{\text {b }}$ & 2.81 & 6.147 & 0.126 & 6.08 \\
\hline Aggregate (Exp.) & 2.53 & & & \\
\hline
\end{tabular}

${ }^{\mathrm{a}}$ Vertical excitation energies and oscillator strengths obtained by TD-DFT/6-31G* calculations in water (C-PCM) on the M06-2Xoptimized geometries

${ }^{b}$ Vertical excitation energies and oscillator strengths obtained by TDA /6-31G* calculations in water (COSMO) on the FF-optimized geometries.

Table 1. Vertical excitation energies in $\mathrm{eV}\left(\mathrm{E}_{\max }\right)$, oscillator strengths, $\mathrm{CT}$ numbers and PR values for the PDI monomer and its aggregates, namely dimer, trimer, tetramer and heptamer. The experimental maximum absorption for PDI aggregate is also reported.

Concerning the excited states topology analysis of dimer, trimer, tetramer, and heptamer the CT values are rather low, especially for the dimer, pointing towards localized Frenkel bright states. Even if the excitation energy of the first bright state is scarcely affected from the considered aggregation geometries (namely the "helix like" or "alternated type") the exciton analysis of the two systems shows 
relevant differences (Table 1). Indeed, when the "alternated type" geometries are considered the charge transfer is strongly reduced (lower CT numbers) while the delocalization of the excitation involves more fragments (higher PR values), with a maximum of 6.0 in the case of the heptamer. This analysis allows quantifying and modeling the effect of the geometrical arrangements on the exciton properties, as well as on the nature of the excited state, even when considering equilibrium geometries, only. On the basis of these findings one could expect a significant impact on the exciton nature and delocalization due to the inclusion of finite temperature effects, as we will discuss in the next sections by analyzing the results of the MD simulations.

\section{Molecular Dynamic Simulations}

Before proceeding with the following analysis, it is useful to remind that, especially in the case of the large-sized aggregates, it is preferable to describe the excited states using the less expensive TDA approach, and to switch from a hybrid functional to a long-range corrected one to avoid the sizeable underestimation of long-range charge-transfer states. As reported in Table S2 in Supplementary Information, we may evidence that, for both B3LYP and CAM-B3LYP, the blue-shift in absorption maxima due to the use of TDA (ca. $0.2-0.3 \mathrm{eV})^{82}$ is, on overall, compensated by the red-shift obtained using the force field-based geometries (ca. $0.2 \mathrm{eV}$ ), the COSMO solvation scheme, and neglecting the terminal chains. Considering, however, that our goal here is getting insights into the nature and delocalization of the exciton, as well as, analyzing the topology of low-energy CT states along the MD simulations, rather than reproducing the position of the experimental absorption maximum of the aggregates, we prefer the use of CAM-B3LYP for the TDA calculations, even if noticeably blue-shifted, as it delivers the right order of bright and CT states. Indeed as pointed out by Dreuw group CAMB3LYP provides better transition densities matrices even if the excitation energies are of lower quality compared to B3LYP. ${ }^{83}$

\subsection{Monomer}


In Figure 4 we report the absorption spectrum of the PDI monomer obtained as the convolution of the vertical transitions obtained from $100 \mathrm{MD}$ snapshots. As compared to the absorption maximum obtained from the static description, and due to the rigidity of the perylene core (Figure S4), the thermal averaged spectrum is almost unaffected, with deviation of less than $0.1 \mathrm{eV}$ for both B3LYP and CAM-B3LYP; however, and as expected, the band is globally broader. Finally, we note that the classing sampling of the ground state conformational shape is not able to reproduce the observed vibronic secondary band, which require the explicit calculation of the Franck-Condon factors ${ }^{36,84}$ to take into account the coupling between electronic and vibrational quantum states.
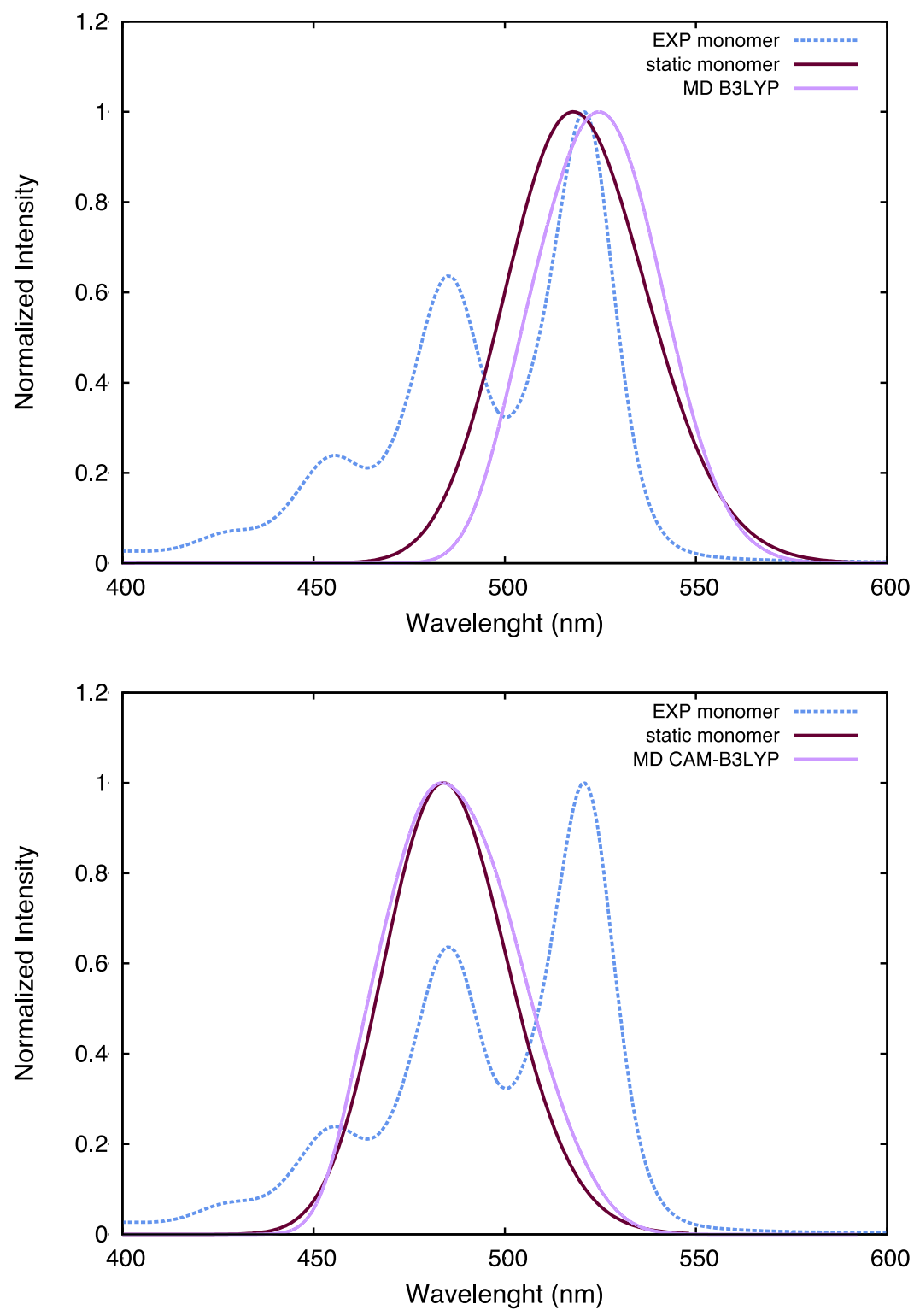
Figure 4. Calculated B3LYP (top panel) and CAM-B3LYP (bottom panel) static (brown full line) and thermal-averaged (violet full line) absorption spectra of the PDI monomer compared to the experimental one (light blue dashed line). A gaussian broadening of $0.1 \mathrm{eV}$ has been used for the convolution.

\subsection{Tetramer}

In Figure 5 we report the distribution of the dihedral angles and of the center of mass distances between consecutive units of the tetrameric aggregates, while the respective time-series are provided in Supporting Information (Figure S5). During the first 100 ns the structure experienced only limited deviations from the helicoidal ideal structure, in the following, however, one can observe an interconversion between the two conformations ("helix like" and "alternated type") proceeding through quite persistent intermediate states (Figure 5).
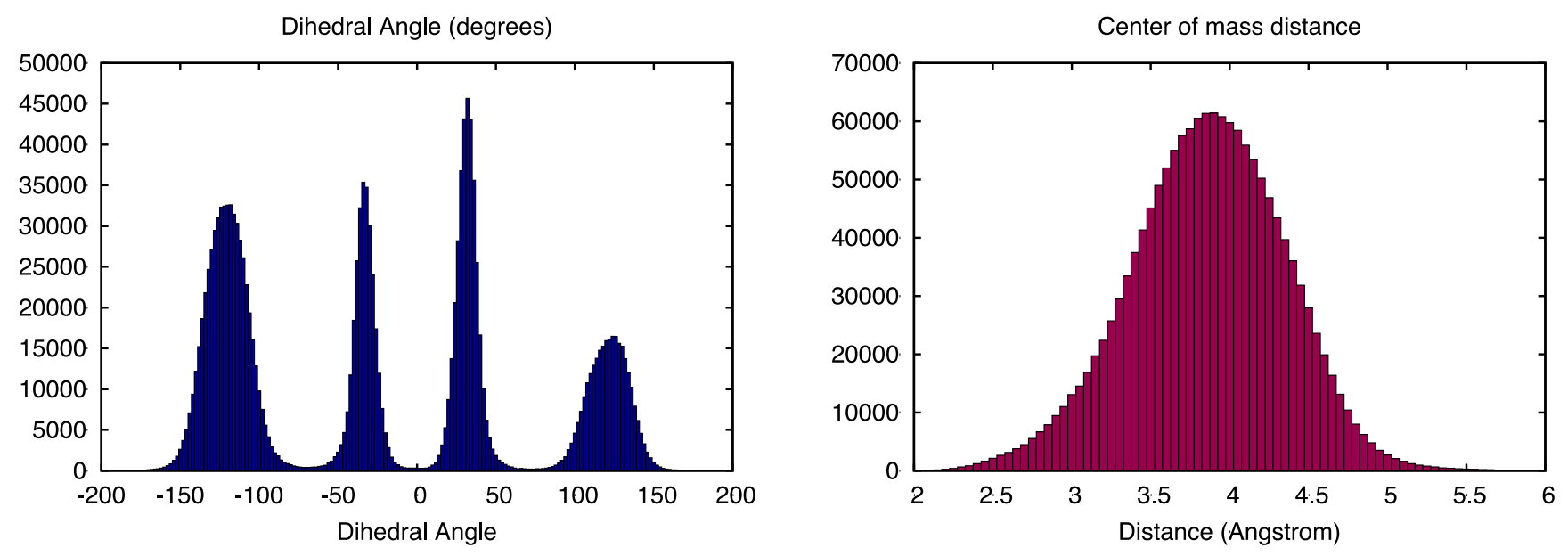

Figure 5. Distributions of the dihedral angle between couple of PDI (left) and of the distance of the center mass of consecutive PDI units (right) obtained from the whole MD production run of the tetramer. Note that the asymmetry with respect to zero can be ascribed to the convergence of the statistical sampling, only. 
On the other hand, and coherently with the high stacking energies (Figure S3), the distance of the center of mass of consecutive units experiences negligible variations along the MD simulation remaining close to $3.5 \AA$.
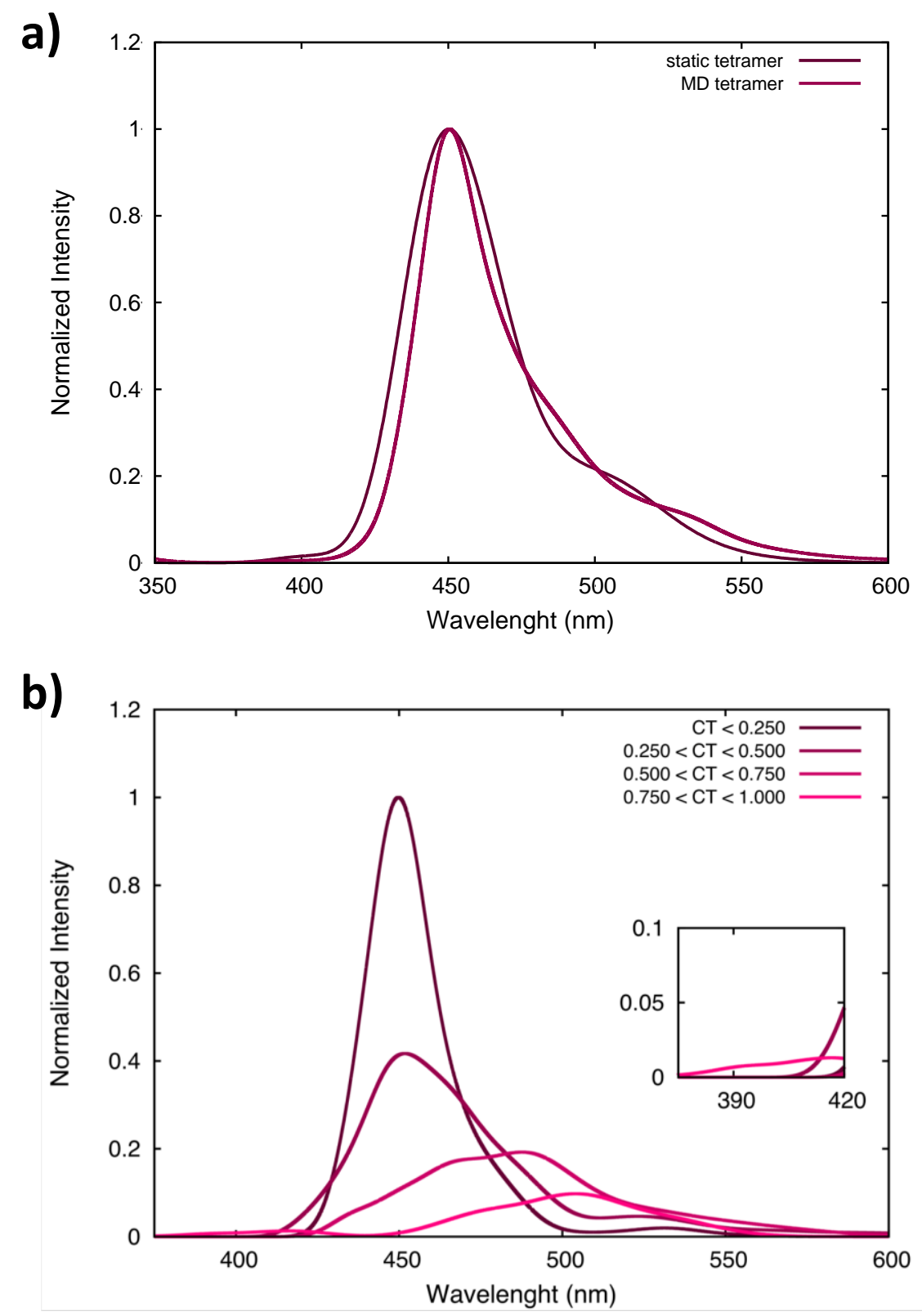

Figure 6. a) Calculated (TDA-DFT CAMB3LYP/6-31G*) thermal-averaged spectra of the PDI tetramer in water (brown) compared to the static one (violet) obtained at the same level of theory on the F.F. minimized structure of the tetramer. b) Decomposition of the calculated spectra taking into accounts only states having specific charge-transfer character (CT index). 
The thermal averaged CAM-B3LYP absorption spectrum is reported in Figure 6, compared to the static one obtained on the F.F. optimized tetramer (top panel). One can note that once again the inclusion of thermal motion effects induces only a negligible red-shift of the absorption maximum. Indeed, as shown for the equilibrium structures, the effect of the two different conformations, i.e. helical or alternated, on the maximum wavelength, is absolutely negligible. However, thermal agitation should be analyzed in terms of the effects it produces on the exciton wavefunction and, in particular, on the exciton delocalization length, that can in turn strongly alter the photophysical properties of the selfaggregates.

To analyze the exciton properties, we computed the CT numbers and the PR values of each excited state on top of the snapshots extracted from the MD trajectory. Their distributions are displayed in Figure 7, while the corresponding time series, are available in Supplementary Information (Figure S6). The distribution of PR values presents a sharp peak at value of 1 , which is indicative of the monomerlike states obtained at high excitation energies. However, the distribution also presents a rather broad but evident maximum peaked around PR of 2.0. Hence, we can see that bimolecular exciton, i.e. excited states delocalized on two PDI units only, are also high probable.
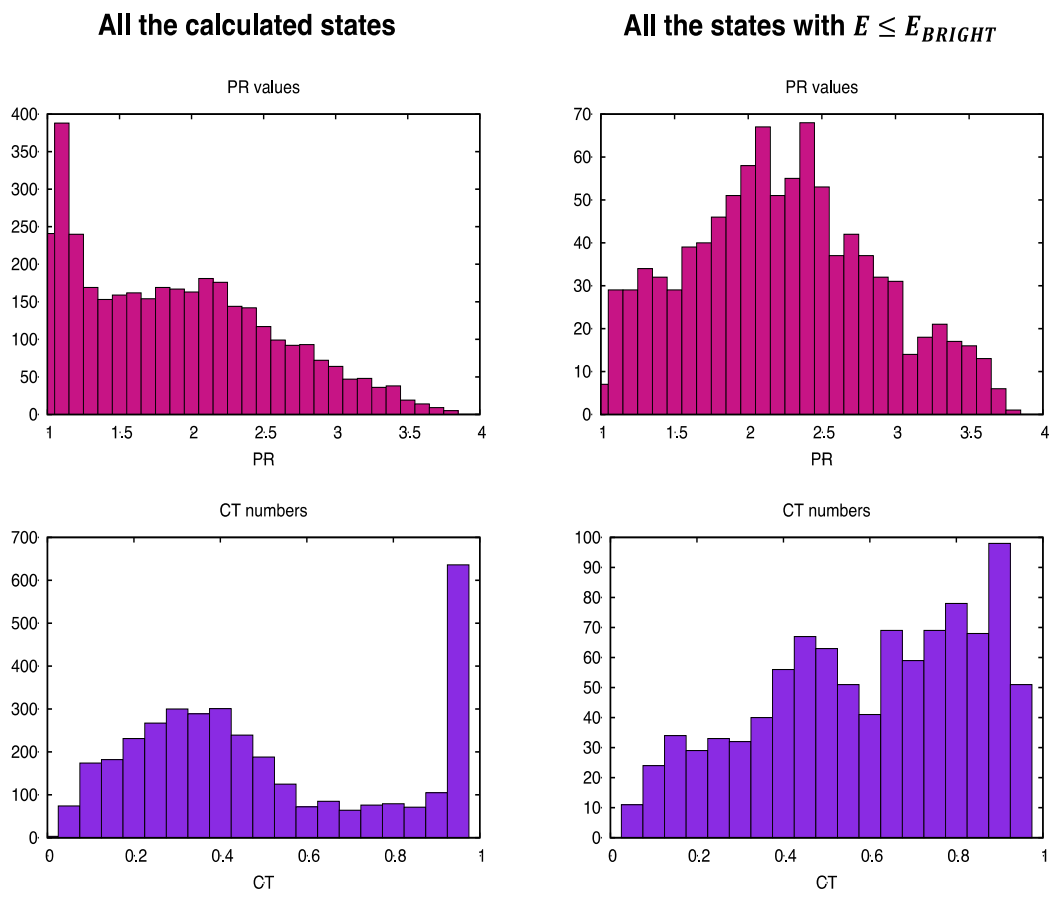
Figure 7. CT numbers and PR values computed from the TDA calculations obtained from MD frames of the PDI tetramer. In the left column are reported the analyses for all the calculated states, in the right column for all the states having an excitation energy equal to or lower than the brightest state.

The PR distribution, however, goes to zero quite rapidly for values greater than 2.5 , and is strictly zero close to 4.0. This aspect implies that only a very few excited states will present excitons delocalized over the whole aggregate and even the ones spread over three units will be quite rare. The topological analysis can be completed taking into account the emergence of charge transfer states (bottom panels in Figure 7). We can observe that most of the states will be quite local, as the broad maximum of the CT distribution centered around 0.3 indicates. However, a number of states characterized by an almost total charge separation (CT ca. 0-9-1.0) appears as indicated by the strongly peaked maximum. Interestingly, even if those highly charge-transfer states will be characterized by very low oscillator strengths they appear at rather small excitation energies, hence they might be populated during the photophysical evolution of the excitonic manifold and could lead to important properties concerning the interfacial charge generation properties of PDI-sensitized devices. Indeed, the accumulation of the CT states at the red-edge of the absorption band can be confirmed by Figure $6 \mathrm{~b}$, in which the calculated spectrum is decomposed in terms of states having specific CT indexes.

\subsection{Heptamer}

Similar considerations also apply for the larger heptamer-PDI self-aggregate, even though the dynamics is obviously more complicated, notably spanning a much more complex conformational space.

The distribution of the dihedral angle between two contiguous units is reported in Figure 8, together with the illustration of some representative snapshots extracted from the MD trajectory, while the corresponding time-series are provided in Supporting Information (Figure S7). The main thermal motion of the PDI units is due to the coupled and simultaneous movement of chunks of monomers, from 

of helical and alternated regions inside the column.
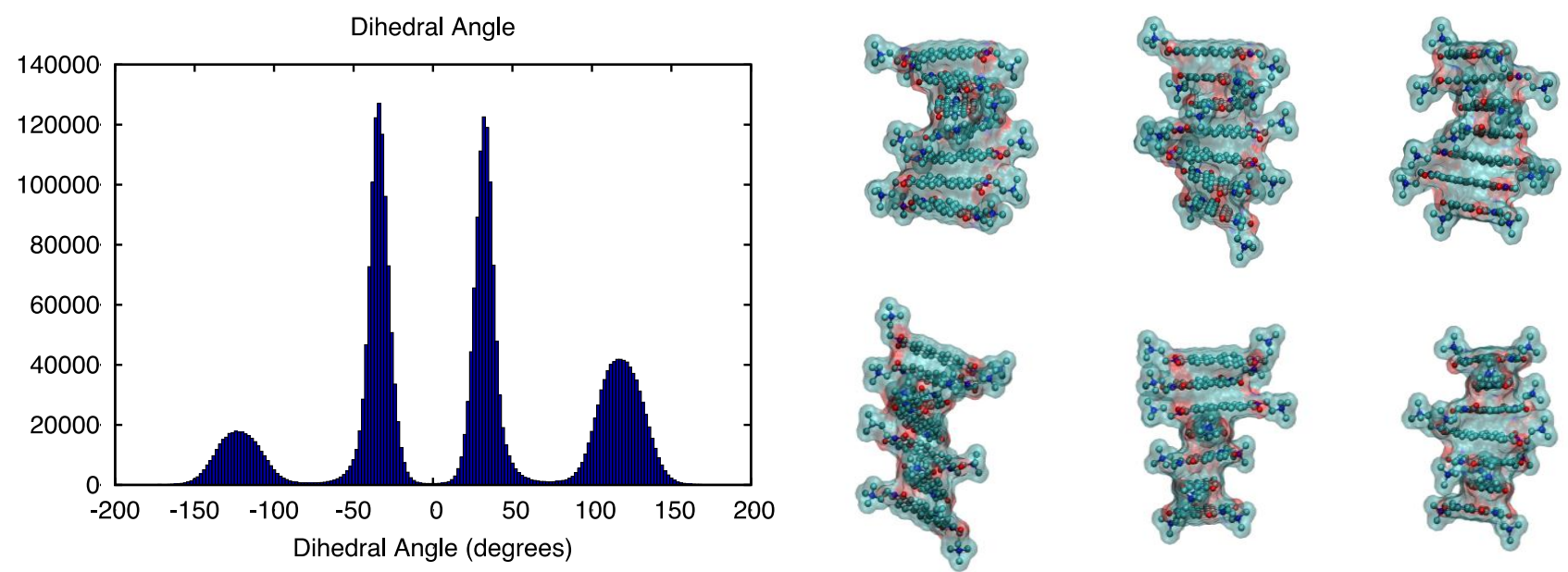

Figure 8. Dihedral angle between each couple of PDI for the heptamer aggregate (left panel) and some representative snapshots extracted along the MD trajectory (right panel).

The absorption spectrum, reported in Figure S8, presents very similar characteristic to the ones evidenced by the tetramer's one. Both the negligible effect of the aggregate size, and the peculiar concerted rotation of chunks of PDI can be rationalized considering the topology of the observed excited states (Figure 9). Indeed the distribution of the PR values presents an important accumulation of entries in the region comprised between 1 and 2, hence suggesting an important presence of monomerlike and bimolecular excitons. Furthermore, and again in agreement with the tetramer case, even if a secondary maximum centered around 3 is evident the distribution goes to zero rapidly for PR values close to 5 . Hence, we can safely conclude that even in the heptamer case only very rare occurrences of exciton length larger than 4-5 units will be observed, while no exciton state delocalized over the entire aggregate can be evidenced. As far as the nature of excited states is concerned, once again very similar considerations to the ones drawn for the tetramer hold, the majority of states, and in particular the brightest one, present a pronounced local character. However, we may still evidence the presence of low energy CT states showing an almost total electron separation involving two PDI units and accumulating 
at the red-edge of the absorption spectrum (Figure S8b). Interestingly, and as evidenced in Figure S9 the exciton localized at the center of the heptamer gives a distribution of PR slightly shifted towards larger values compared to the global one, while the amount of charge-transfer is reduced. Hence, due to its less pronounced flexibility the central units of the column will be characterized by the largest exciton-length, while the edge-monomer will experience a higher influence of charge-transfer states.

Overall, our findings are consistent with the experimental conclusions drawn by Kim and coworkers $^{64}$ on perylene bisimides helices composed of 10 units, indicating an initial delocalization of the Frenkel-type exciton on 3 monomeric units. Indeed, our results confirm that due to the combined effect of electronic and thermal factors PDI are unable to produce strongly delocalized excitons, as a consequence the most probable coherent electron or energy transfer phenomena, will involve at maximum 4-5 units. Hence the preparation of larger-size $\pi$-stacked aggregates will most probably not be strongly beneficial in enhancing PDI photophysical and charge transport properties.

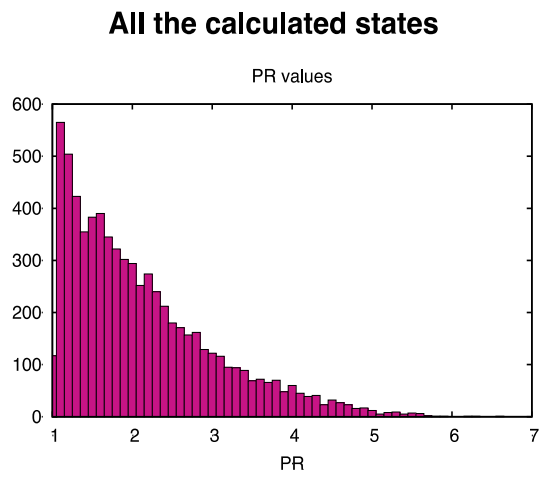

All the states with $E \leq E_{B R I G H T}$
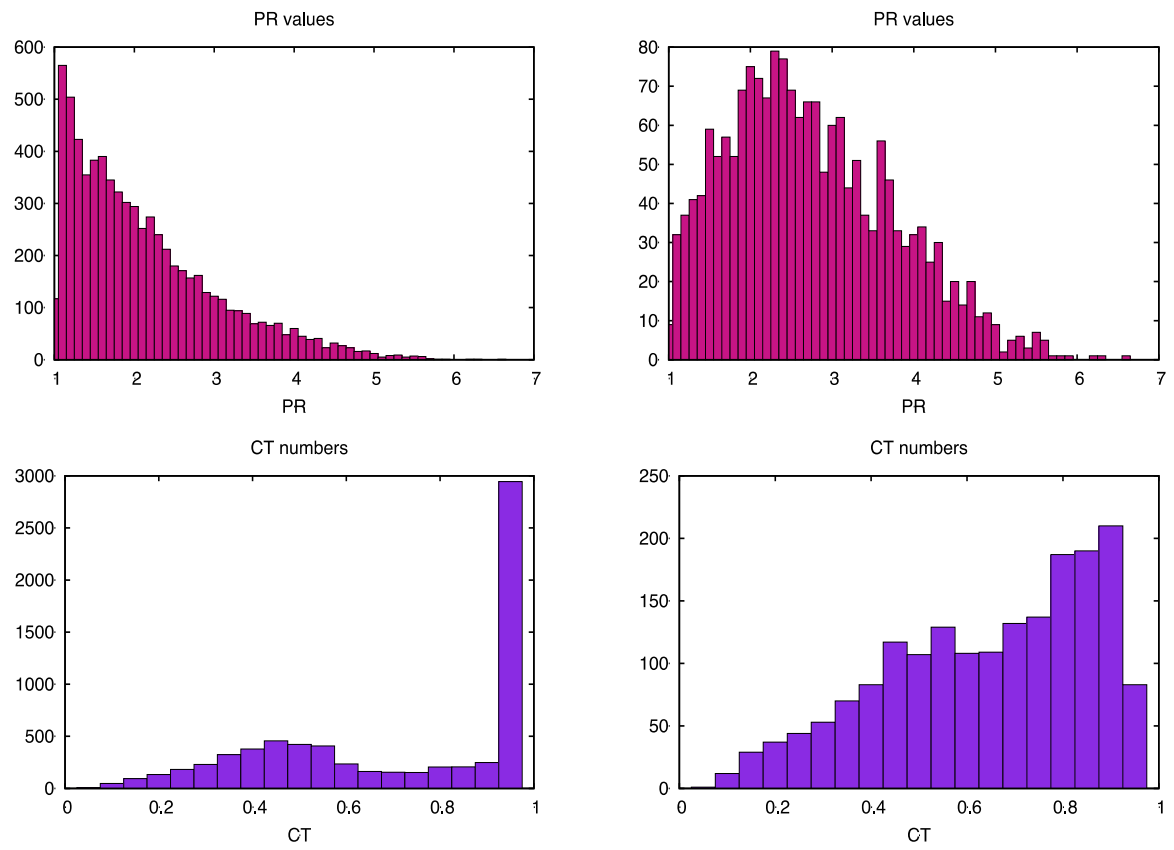
Figure 9. CT numbers and Partecipation Ratio (PR) values computed from the TDA calculations obtained from MD frames of the PDI hepatmer. In the left column are reported the analyses for all the calculated states, in the right column for all the states having an excitation energy equal to or lower than the brightest state.

\section{Conclusions}

Using a combination of quantum chemistry methods and classical molecular mechanics we have deeply analyzed the optical properties of PDI self-aggregates that have recently been used as light-harvesting systems for water splitting devices or solar cells. ${ }^{33-35}$ In particular we have confirmed the capacity of PDI $\pi$-stacked $\mathrm{H}$-aggregates to give raise to delocalized excitonic wavefunctions. The effects of thermal motion on the shape and position of the absorption spectrum are, however, negligible, both in the case of the monomer and of the aggregates. Even if not evident in the optical properties, the effect of thermal motion is crucial in dictating the global properties of the aggregates excited states manifold, in particular even in the case of longer helices the exciton delocalization almost never exceeds 5 PDI units, confirming recent experimental observations. ${ }^{64}$ This aspect is also reflected in the peculiar large scale motion observed in the aggregate MD and characterized by the concerted and simultaneous rotation of chunks of the PDI column. Even if the delocalization of the Frenkel exciton state is, however, not extremely large compared to similar self-aggregate materials, one should evidence the presence of dark, but low-lying, charge-transfer excited state leading to a full electron-hole separation between two units that, hence, can be efficiently exploited to optimize charge separation and transport in PDI-based optical devices.

\section{Supporting Information Available}

The $\mathrm{E}_{0-0}$ and the vertical excitation energies for PDI monomer considering different functionals. 
The analyses of the MD trajectory of the PDI monomer and aggregates. The relaxed dihedral angle scan for PDI dimer. The comparison between the excited state energies obtained from the DFT geometries and the force field geometries. The Thermal-averaged absorption spectra of the PDI tetramer and heptamer. The topological analysis of the heptamer excited state centered on the central PDI units, only. This material is available free of charge via the Internet at http://pubs.acs.org.

\section{Aknowlwdgements}

We thank ANR JCJC HELIOSH2 (ANR-17-CE05-0007-01) for financial support and HPC resources from the mésocentre EXPLOR of Université de Lorraine (Project 2018CPMXX0602), and from the LPCT local computing clusters.

\section{References:}

(1) Reineke, S.; Thomschke, M.; Lüssem, B.; Leo, K. White Organic Light-Emitting Diodes: Status and Perspective. Rev. Mod. Phys. 2013, 85 (3), 1245-1293.

(2) Reineke, S. Complementary LED Technologies. Nat. Mater. 2015, 14, 459.

(3) Agrawal, S.; Leijtens, T.; Ronca, E.; Pastore, M.; Snaith, H.; De Angelis, F. Modeling the Effect of Ionic Additives on the Optical and Electronic Properties of a Dye-Sensitized TiO2 Heterointerface: Absorption, Charge Injection and Aggregation. J. Mater. Chem. A 2013, 1 (46), $14675-14685$.

(4) Clarke, T. M.; Durrant, J. R. Charge Photogeneration in Organic Solar Cells. Chem. Rev. 2010, $110(11), 6736-6767$.

(5) Ostroverkhova, O. Organic Optoelectronic Materials: Mechanisms and Applications. Chem. Rev. 2016, $116(22), 13279-13412$. 
(6) Kasha, M. Energy Transfer Mechanisms and the Molecular Exciton Model for Molecular Aggregates. Radiat. Res. 1963, 20 (1), 55-70.

(7) Hochstrasser, R. M.; Kasha, M. Application of the Exciton Model to Mono-Molecular Lamellar Systems. Photochem. Photobiol. 1964, 3 (4), 317-331.

(8) Eisele, D. M.; Knoester, J.; Kirstein, S.; Rabe, J. P.; Vanden Bout, D. A. Uniform Exciton Fluorescence from Individual Molecular Nanotubes Immobilized on Solid Substrates. Nat. Nanotechnol. 2009, 4, 658.

(9) Hestand, N. J.; Spano, F. C. Molecular Aggregate Photophysics beyond the Kasha Model: Novel Design Principles for Organic Materials. Acc. Chem. Res. 2017, 50 (2), 341-350.

(10) Hestand, N. J.; Spano, F. C. Expanded Theory of H- and J-Molecular Aggregates: The Effects of Vibronic Coupling and Intermolecular Charge Transfer. Chem. Rev. 2018, 118 (15), 7069-7163.

(11) O’Regan, B.; Grätzel, M. A Low-Cost, High-Efficiency Solar Cell Based on Dye-Sensitized Colloidal TiO2 Films. Nature 1991, 353 (6346), 737-740.

(12) Hagfeldt, A.; Boschloo, G.; Sun, L.; Kloo, L.; Pettersson, H. Dye-Sensitized Solar Cells. Chem. Rev. 2010, $110(11), 6595-6663$.

(13) Monti, S.; Pastore, M.; Li, C.; De Angelis, F.; Carravetta, V. Theoretical Investigation of Adsorption, Dynamics, Self-Aggregation, and Spectroscopic Properties of the D102 Indoline Dye on an Anatase (101) Substrate. J. Phys. Chem. C 2016, 120 (5), 2787-2796.

(14) Yu, Z.; Li, F.; Sun, L. Recent Advances in Dye-Sensitized Photoelectrochemical Cells for Solar Hydrogen Production Based on Molecular Components. Energy Environ. Sci. 2015, 8 (3), 760775.

(15) Vaissier, V.; Mosconi, E.; Moia, D.; Pastore, M.; Frost, J. M.; De Angelis, F.; Barnes, P. R. F.; 
Nelson, J. Effect of Molecular Fluctuations on Hole Diffusion within Dye Monolayers. Chem.

Mater. 2014, 26 (16), 4731-4740.

(16) Pastore, M.; Etienne, T.; De Angelis, F. Structural and Electronic Properties of Dye-Sensitized TiO2 for Solar Cell Applications: From Single Molecules to Self-Assembled Monolayers. $J$.

Mater. Chem. C 2016, 4 (20), 4346-4373.

(17) Pastore, M.; De Angelis, F. Aggregation of Organic Dyes on TiO2 in Dye-Sensitized Solar Cells Models: An Ab Initio Investigation. ACS Nano 2010, 4 (1), 556-562.

(18) Brennaman, M. K.; Dillon, R. J.; Alibabaei, L.; Gish, M. K.; Dares, C. J.; Ashford, D. L.; House, R. L.; Meyer, G. J.; Papanikolas, J. M.; Meyer, T. J. Finding the Way to Solar Fuels with DyeSensitized Photoelectrosynthesis Cells. J. Am. Chem. Soc. 2016, 138 (40), 13085-13102.

(19) Pastore, M. First Principle Modelling of Materials and Processes in Dye-Sensitized Photoanodes for Solar Energy and Solar Fuels. Computation . 2017.

(20) Xu, P.; McCool, N. S.; Mallouk, T. E. Water Splitting Dye-Sensitized Solar Cells. Nano Today 2017, 14, 42-58.

(21) Horiuchi, T.; Miura, H.; Uchida, S. Highly-Efficient Metal-Free Organic Dyes for Dye-Sensitized Solar Cells. Chem. Commun. 2003, No. 24, 3036-3037.

(22) Agrawal, S.; Pastore, M.; Marotta, G.; Reddy, M. A.; Chandrasekharam, M.; De Angelis, F. Optical Properties and Aggregation of Phenothiazine-Based Dye-Sensitizers for Solar Cells Applications: A Combined Experimental and Computational Investigation. J. Phys. Chem. C 2013, 117 (19), 9613-9622.

(23) Tatay, S.; Haque, S. A.; O’Regan, B.; Durrant, J. R.; Verhees, W. J. H.; Kroon, J. M.; VidalFerran, A.; Gaviña, P.; Palomares, E. Kinetic Competition in Liquid Electrolyte and Solid-State Cyanine Dye Sensitized Solar Cells. J. Mater. Chem. 2007, 17 (29), 3037-3044. 
(24) Wang, Z.-S.; Cui, Y.; Dan-oh, Y.; Kasada, C.; Shinpo, A.; Hara, K. Thiophene-Functionalized Coumarin Dye for Efficient Dye-Sensitized Solar Cells: Electron Lifetime Improved by Coadsorption of Deoxycholic Acid. J. Phys. Chem. C 2007, 111 (19), 7224-7230.

(25) Horiuchi, T.; Miura, H.; Sumioka, K.; Uchida, S. High Efficiency of Dye-Sensitized Solar Cells Based on Metal-Free Indoline Dyes. J. Am. Chem. Soc. 2004, 126 (39), 12218-12219.

(26) Heo, J. H.; Im, S. H.; Noh, J. H.; Mandal, T. N.; Lim, C.-S.; Chang, J. A.; Lee, Y. H.; Kim, H.; Sarkar, A.; Nazeeruddin, M. K.; et al. Efficient Inorganic-Organic Hybrid Heterojunction Solar Cells Containing Perovskite Compound and Polymeric Hole Conductors. Nat. Photonics 2013, 7 , 486.

(27) Chandiran, A. K.; Zakeeruddin, S. M.; Humphry-Baker, R.; Nazeeruddin, M. K.; Grätzel, M.; Sauvage, F. Investigation on the Interface Modification of TiO2 Surfaces by Functional CoAdsorbents for High-Efficiency Dye-Sensitized Solar Cells. ChemPhysChem 2017, 18 (19), $2724-2731$.

(28) Jungsuttiwong, S.; Sirithip, K.; Prachumrak, N.; Tarsang, R.; Sudyoadsuk, T.; Namuangruk, S.; Kungwan, N.; Promarak, V.; Keawin, T. Significant Enhancement in the Performance of Porphyrin for Dye-Sensitized Solar Cells: Aggregation Control Using Chenodeoxycholic Acid. New J. Chem. 2017, 41 (15), 7081-7091.

(29) Ehret, A.; Stuhl, L.; Spitler, M. T. Spectral Sensitization of TiO2 Nanocrystalline Electrodes with Aggregated Cyanine Dyes. J. Phys. Chem. B 2001, 105 (41), 9960-9965.

(30) Sayama, K.; Tsukagoshi, S.; Hara, K.; Ohga, Y.; Shinpou, A.; Abe, Y.; Suga, S.; Arakawa, H. Photoelectrochemical Properties of J Aggregates of Benzothiazole Merocyanine Dyes on a Nanostructured TiO2 Film. J. Phys. Chem. B 2002, 106 (6), 1363-1371.

(31) Khazraji, A. C.; Hotchandani, S.; Das, S.; Kamat, P. V. Controlling Dye (Merocyanine-540) 
Aggregation on Nanostructured TiO2 Films. An Organized Assembly Approach for Enhancing the Efficiency of Photosensitization. J. Phys. Chem. B 1999, 103 (22), 4693-4700.

(32) Marotta, G.; Reddy, M. A.; Singh, S. P.; Islam, A.; Han, L.; De Angelis, F.; Pastore, M.;

Chandrasekharam, M. Novel Carbazole-Phenothiazine Dyads for Dye-Sensitized Solar Cells: A Combined Experimental and Theoretical Study. ACS Appl. Mater. Interfaces 2013, 5 (19), 96359647.

(33) Ronconi, F.; Syrgiannis, Z.; Bonasera, A.; Prato, M.; Argazzi, R.; Caramori, S.; Cristino, V.; Bignozzi, C. A. Modification of Nanocrystalline WO3 with a Dicationic Perylene Bisimide: Applications to Molecular Level Solar Water Splitting. J. Am. Chem. Soc. 2015, 137 (14), 46304633.

(34) Berardi, S.; Cristino, V.; Canton, M.; Boaretto, R.; Argazzi, R.; Benazzi, E.; Ganzer, L.; Borrego Varillas, R.; Cerullo, G.; Syrgiannis, Z.; et al. Perylene Diimide Aggregates on Sb-Doped SnO2: Charge Transfer Dynamics Relevant to Solar Fuel Generation. J. Phys. Chem. C 2017, 121 (33), $17737-17745$.

(35) Bonchio, M.; Syrgiannis, Z.; Burian, M.; Marino, N.; Pizzolato, E.; Dirian, K.; Rigodanza, F.; Volpato, G. A.; La Ganga, G.; Demitri, N.; et al. Hierarchical Organization of Perylene Bisimides and Polyoxometalates for Photo-Assisted Water Oxidation. Nat. Chem. 2018, doi: 10.1038/s41557-018-0172-y.

(36) Clark, A. E.; Qin, C.; Li, A. D. Q. Beyond Exciton Theory: A Time-Dependent DFT and Franck-Condon Study of Perylene Diimide and Its Chromophoric Dimer. J. Am. Chem. Soc. 2007, 129 (24), 7586-7595.

(37) Houari, Y.; Laurent, A. D.; Jacquemin, D. Spectral Signatures of Perylene Diimide Derivatives: Insights From Theory. J. Phys. Chem. C 2013, 117 (42), 21682-21691. 
(38) Kozma, E.; Catellani, M. Perylene Diimides Based Materials for Organic Solar Cells. Dye. Pigment. 2013, 98 (1), 160-179.

(39) Huang, C.; Barlow, S.; Marder, S. R. Perylene-3,4,9,10-Tetracarboxylic Acid Diimides: Synthesis, Physical Properties, and Use in Organic Electronics. J. Org. Chem. 2011, 76 (8), $2386-2407$.

(40) Li, C.; Wonneberger, H. Perylene Imides for Organic Photovoltaics: Yesterday, Today, and Tomorrow. Adv. Mater. 2012, 24 (5), 613-636.

(41) Hanson, K.; Brennaman, M. K.; Luo, H.; Glasson, C. R. K.; Concepcion, J. J.; Song, W.; Meyer, T. J. Photostability of Phosphonate-Derivatized, RuII Polypyridyl Complexes on Metal Oxide Surfaces. ACS Appl. Mater. Interfaces 2012, 4 (3), 1462-1469.

(42) Kamire, R. J.; Materna, K. L.; Hoffeditz, W. L.; Phelan, B. T.; Thomsen, J. M.; Farha, O. K.; Hupp, J. T.; Brudvig, G. W.; Wasielewski, M. R. Photodriven Oxidation of Surface-Bound Iridium-Based Molecular Water-Oxidation Catalysts on Perylene-3,4-Dicarboximide-Sensitized TiO2 Electrodes Protected by an A12O3 Layer. J. Phys. Chem. C 2017, 121 (7), 3752-3764.

(43) Brennaman, M. K.; Norris, M. R.; Gish, M. K.; Grumstrup, E. M.; Alibabaei, L.; Ashford, D. L.; Lapides, A. M.; Papanikolas, J. M.; Templeton, J. L.; Meyer, T. J. Ultrafast, Light-Induced Electron Transfer in a Perylene Diimide Chromophore-Donor Assembly on TiO2. J. Phys. Chem. Lett. 2015, 6 (23), 4736-4742.

(44) Pastore, M.; De Angelis, F. First-Principles Modeling of a Dye-Sensitized TiO2/IrO2 Photoanode for Water Oxidation. J. Am. Chem. Soc. 2015, 137 (17), 5798-5809.

(45) Lasser, L.; Ronca, E.; Pastore, M.; De Angelis, F.; Cornil, J.; Lazzaroni, R.; Beljonne, D. Energy Level Alignment at Titanium Oxide-Dye Interfaces: Implications for Electron Injection and Light Harvesting. J. Phys. Chem. C 2015, 119 (18), 9899-9909. 
(46) Guo, Q.; Xu, Y.; Xiao, B.; Zhang, B.; Zhou, E.; Wang, F.; Bai, Y.; Hayat, T.; Alsaedi, A.; Tan, Z. Effect of Energy Alignment, Electron Mobility, and Film Morphology of Perylene Diimide Based Polymers as Electron Transport Layer on the Performance of Perovskite Solar Cells. ACS Appl. Mater. Interfaces 2017, 9 (12), 10983-10991.

(47) Settels, V.; Schubert, A.; Tafipolski, M.; Liu, W.; Stehr, V.; Topczak, A. K.; Pflaum, J.; Deibel, C.; Fink, R. F.; Engel, V.; et al. Identification of Ultrafast Relaxation Processes As a Major Reason for Inefficient Exciton Diffusion in Perylene-Based Organic Semiconductors. J. Am. Chem. Soc. 2014, $136(26), 9327-9337$.

(48) Menke, S. M.; Luhman, W. A.; Holmes, R. J. Tailored Exciton Diffusion in Organic Photovoltaic Cells for Enhanced Power Conversion Efficiency. Nat. Mater. 2012, 12, 152.

(49) Sung, J.; Nowak-Król, A.; Schlosser, F.; Fimmel, B.; Kim, W.; Kim, D.; Würthner, F. Direct Observation of Excimer-Mediated Intramolecular Electron Transfer in a Cofacially-Stacked Perylene Bisimide Pair. J. Am. Chem. Soc. 2016, 138 (29), 9029-9032.

(50) Kennehan, E. R.; Grieco, C.; Brigeman, A. N.; Doucette, G. S.; Rimshaw, A.; Bisgaier, K.; Giebink, N. C.; Asbury, J. B. Using Molecular Vibrations to Probe Exciton Delocalization in Films of Perylene Diimides with Ultrafast Mid-IR Spectroscopy. Phys. Chem. Chem. Phys. 2017, 19 (36), 24829-24839.

(51) Fennel, F.; Wolter, S.; Xie, Z.; Plötz, P.-A.; Kühn, O.; Würthner, F.; Lochbrunner, S. Biphasic Self-Assembly Pathways and Size-Dependent Photophysical Properties of Perylene Bisimide Dye Aggregates. J. Am. Chem. Soc. 2013, 135 (50), 18722-18725.

(52) Ye, T.; Singh, R.; Butt, H.-J.; Floudas, G.; Keivanidis, P. E. Effect of Local and Global Structural Order on the Performance of Perylene Diimide Excimeric Solar Cells. ACS Appl. Mater. Interfaces 2013, 5 (22), 11844-11857. 
(53) Walsh, J. J.; Lee, J. R.; Draper, E. R.; King, S. M.; Jäckel, F.; Zwijnenburg, M. A.; Adams, D. J.; Cowan, A. J. Controlling Visible Light Driven Photoconductivity in Self-Assembled Perylene Bisimide Structures. J. Phys. Chem. C 2016, 120 (33), 18479-18486.

(54) Kaufmann, C.; Kim, W.; Nowak-Król, A.; Hong, Y.; Kim, D.; Würthner, F. Ultrafast Exciton Delocalization, Localization, and Excimer Formation Dynamics in a Highly Defined Perylene Bisimide Quadruple $\pi$-Stack. J. Am. Chem. Soc. 2018, 140 (12), 4253-4258.

(55) Zhao, H.-M.; Pfister, J.; Settels, V.; Renz, M.; Kaupp, M.; Dehm, V. C.; Würthner, F.; Fink, R. F.; Engels, B. Understanding Ground- and Excited-State Properties of Perylene Tetracarboxylic Acid Bisimide Crystals by Means of Quantum Chemical Computations. J. Am. Chem. Soc. 2009, $131(43), 15660-15668$.

(56) Yoo, H.; Yang, J.; Yousef, A.; Wasielewski, M. R.; Kim, D. Excimer Formation Dynamics of Intramolecular $\pi$-Stacked Perylenediimides Probed by Single-Molecule Fluorescence Spectroscopy. J. Am. Chem. Soc. 2010, 132 (11), 3939-3944.

(57) Fink, R. F.; Seibt, J.; Engel, V.; Renz, M.; Kaupp, M.; Lochbrunner, S.; Zhao, H.-M.; Pfister, J.; Würthner, F.; Engels, B. Exciton Trapping in $\pi$-Conjugated Materials: A Quantum-ChemistryBased Protocol Applied to Perylene Bisimide Dye Aggregates. J. Am. Chem. Soc. 2008, 130 (39), $12858-12859$.

(58) Engel, E.; Schmidt, K.; Beljonne, D.; Brédas, J.-L.; Assa, J.; Fröb, H.; Leo, K.; Hoffmann, M. Transient Absorption Spectroscopy and Quantum-Chemical Studies of Matrix-Isolated Perylene Derivatives. Phys. Rev. B 2006, 73 (24), 245216.

(59) Atilgan, S.; Ozdemir, T.; Akkaya, E. U. Selective Hg(II) Sensing with Improved Stokes Shift by Coupling the Internal Charge Transfer Process to Excitation Energy Transfer. Org. Lett. 2010, 12 (21), 4792-4795. 
(60) Chen, Z.; Stepanenko, V.; Dehm, V.; Prins, P.; Siebbeles, L. D. A.; Seibt, J.; Marquetand, P.; Engel, V.; Würthner, F. Photoluminescence and Conductivity of Self-Assembled $\pi-\pi$ Stacks of Perylene Bisimide Dyes. Chem. - A Eur. J. 2007, 13 (2), 436-449.

(61) Würthner, F.; Chen, Z.; Dehm, V.; Stepanenko, V. One-Dimensional Luminescent Nanoaggregates of Perylene Bisimides. Chem. Commun. 2006, No. 11, 1188-1190.

(62) Spano, F. C.; Meskers, S. C. J.; Hennebicq, E.; Beljonne, D. Probing Excitation Delocalization in Supramolecular Chiral Stacks by Means of Circularly Polarized Light: Experiment and Modeling. J. Am. Chem. Soc. 2007, 129 (22), 7044-7054.

(63) Settels, V.; Liu, W.; Pflaum, J.; Fink, R. F.; Engels, B. Comparison of the Electronic Structure of Different Perylene-Based Dye-Aggregates. J. Comput. Chem. 2012, 33 (18), 1544-1553.

(64) Sung, J.; Kim, P.; Fimmel, B.; Würthner, F.; Kim, D. Direct Observation of Ultrafast Coherent Exciton Dynamics in Helical $\pi$-Stacks of Self-Assembled Perylene Bisimides. Nat. Commun. 2015, 6,8646 .

(65) Spano, F. C.; Silva, C. H- and J-Aggregate Behavior in Polymeric Semiconductors. Annu. Rev. Phys. Chem. 2014, 65 (1), 477-500.

(66) Walker, M.; Harvey, A. J. A.; Sen, A.; Dessent, C. E. H. Performance of M06, M06-2X, and M06-HF Density Functionals for Conformationally Flexible Anionic Clusters: M06 Functionals Perform Better than B3LYP for a Model System with Dispersion and Ionic Hydrogen-Bonding Interactions. J. Phys. Chem. A 2013, 117 (47), 12590-12600.

(67) Tomasi, J.; Mennucci, B.; Cammi, R. Quantum Mechanical Continuum Solvation Models. Chem. Rev. 2005, 105 (8), 2999-3093.

(68) Frisch, M. J.; Trucks, G. W.; Schlegel, H. B.; Scuseria, G. E.; Robb, M. A.; Cheeseman, J. R.; Scalmani, G.; Barone, V.; Petersson, G. A.; Nakatsuji, H.; et al. Gaussian 09, Revision D.01. 
Gaussian 09, Revis. D.01, Gaussian, Inc., Wallingford CT, 2013.

(69) Wang, J.; Wolf, R. M.; Caldwell, J. W.; Kollman, P. A.; Case, D. A. Development and Testing of a General Amber Force Field. J. Comput. Chem. 2004, 25 (9), 1157-1174.

(70) Mark, P.; Nilsson, L. Structure and Dynamics of the TIP3P, SPC, and SPC/E Water Models at 298 K. J. Phys. Chem. A 2001, 105 (43), 9954-9960.

(71) Case, D. A.; Betz, R. M.; Cerutti, D. S.; T.E. Cheatham, I.; Darden, T. A.; Duke, R. E.; Giese, T. J.; Gohlke, H.; Goetz, A. W.; Homeyer, N.; et al. No Title. AMBER 2016, Univ. California, San Fr.

(72) Titov, A. V.; Ufimtsev, I. S.; Luehr, N.; Martinez, T. J. Generating Efficient Quantum Chemistry Codes for Novel Architectures. J. Chem. Theory Comput. 2013, 9 (1), 213-221.

(73) Yanai, T.; Tew, D. P.; Handy, N. C. A New Hybrid Exchange-Correlation Functional Using the Coulomb-Attenuating Method (CAM-B3LYP). Chem. Phys. Lett. 2004, 393 (1-3), 51-57.

(74) Klamt, A.; Schüürmann, G. COSMO: A New Approach to Dielectric Screening in Solvents with Explicit Expressions for the Screening Energy and Its Gradient. J. Chem. Soc., Perkin Trans. 2 1993, No. 5, 799-805.

(75) Plasser, F.; Wormit, M.; Dreuw, A. New Tools for the Systematic Analysis and Visualization of Electronic Excitations. I. Formalism. J. Chem. Phys. 2014, 141 (2), 24106.

(76) Plasser, F.; Lischka, H. Analysis of Excitonic and Charge Transfer Interactions from Quantum Chemical Calculations. J. Chem. Theory Comput. 2012, 8 (8), 2777-2789.

(77) Plasser, F.; Bäppler, S. A.; Wormit, M.; Dreuw, A. New Tools for the Systematic Analysis and Visualization of Electronic Excitations. II. Applications. J. Chem. Phys. 2014, 141 (2), 24107.

(78) Delgado, M. C. R.; Kim, E.-G.; Filho, D. A. da S.; Bredas, J.-L. Tuning the Charge-Transport 
Parameters of Perylene Diimide Single Crystals via End and/or Core Functionalization: A Density Functional Theory Investigation. J. Am. Chem. Soc. 2010, 132 (10), 3375-3387.

(79) Ghosh, S.; Li, X.-Q.; Stepanenko, V.; Würthner, F. Control of H- and J-Type $\pi$ Stacking by Peripheral Alkyl Chains and Self-Sorting Phenomena in Perylene Bisimide Homo- and Heteroaggregates. Chem. - A Eur. J. 2008, 14 (36), 11343-11357.

(80) Siddiqui, S.; Spano, F. C. H- and J-Aggregates of Conjugated Polymers and Oligomers: A Theoretical Investigation. Chem. Phys. Lett. 1999, 308 (1), 99-105.

(81) Nogueira, J. J.; Plasser, F.; González, L. Electronic Delocalization, Charge Transfer and Hypochromism in the UV Absorption Spectrum of Polyadenine Unravelled by Multiscale Computations and Quantitative Wavefunction Analysis. Chem. Sci. 2017, 8 (8), 5682-5691.

(82) Charaf-Eddin, A.; Planchat, A.; Mennucci, B.; Adamo, C.; Jacquemin, D. Choosing a Functional for Computing Absorption and Fluorescence Band Shapes with TD-DFT. J. Chem. Theory Comput. 2013, 9 (6), 2749-2760.

(83) Mewes, S. A.; Plasser, F.; Krylov, A.; Dreuw, A. Benchmarking Excited-State Calculations Using Exciton Properties. J. Chem. Theory Comput. 2018, 14 (2), 710-725.

(84) Santoro, F.; Improta, R.; Lami, A.; Bloino, J.; Barone, V. Effective Method to Compute FranckCondon Integrals for Optical Spectra of Large Molecules in Solution. J. Chem. Phys. 2007, 126 (8), 084509. 


\section{TOC Graphics}

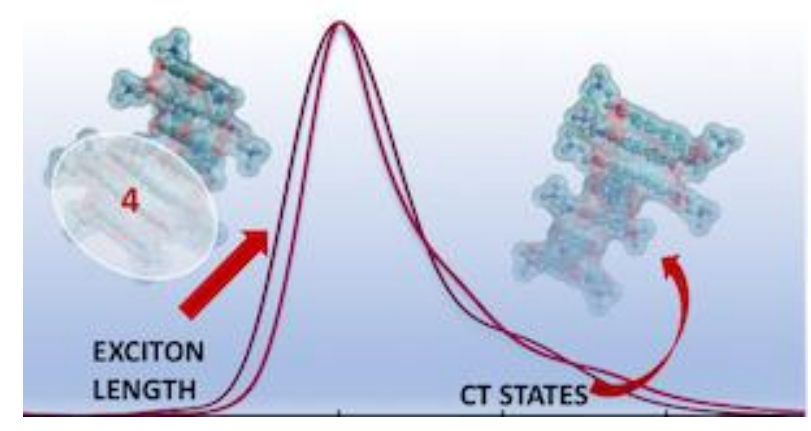

\section{Invited author's biography}

Dr. Mariachiara Pastore is researcher at the French National Centre for Scientific Research (CNRS) in the Laboratoire de Physique et chimie Théoriques, Université de Lorraine, Nancy, France. She was the recipient of the "Carla Roetti" prize 2014 from the Italian Chemistry Society (SCI), dedicated to theoretical and computational chemists under 40 and of the Ricercat@mente award in 2013 from the Italian National Research Council (CNR) for the Best Young Researcher under 35 years old in Chemical Science and Materials Technology. She is an expert in the development and application of ab initio methods to the modelling of the structural, electronic and optical properties of organic/inorganic hybrid materials and interfaces in the broad field of solar energy and solar fuels devices. Her research interests span from the development and application of highly correlated $a b$ initio methods (multireference perturbation theories) and hybrid density functional theory/wavefunction-based methods to the study of excited states in small and medium size molecules, to the characterization of the optical and charge separation properties in molecule/semiconductor interfaces.

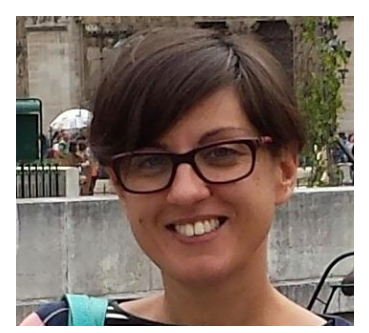

